\title{
Remodeling of the Conformational Dynamics of Noncanonical DNA Structures by Monomeric and Aggregated $\alpha$-Synuclein
}

\author{
Jim-Marcel Knop ${ }^{\ddagger}$,Sanjib K. Mukherjee ${ }^{\ddagger}$ Rosario Oliva, Simone Möbitz, and Roland Winter* \\ Physical Chemistry I - Biophysical Chemistry, Faculty of Chemistry and Chemical Biology, TU Dortmund University, Otto- \\ Hahn Street 4a, 44227 Dortmund, Germany
}

\section{Supporting Information}

Table of Contents

S.1 Experimental and Methods Section

S.2 Supporting References

S.3 Supporting Figures

\section{S.1 Experimental and Methods Section}

Materials and sample preparation

Ficoll 70 was purchased from Carl Roth $\mathrm{GmbH}+\mathrm{Co} . \mathrm{KG}$ (Karlsruhe, Germany) and was used as received. As described by Baltierra-Jasso et al., ${ }^{1}$ the DNA oligonucleotides were synthesized and fluorescently labeled by IBA life solutions GmbH (Goettingen, Germany). The sequences of these oligonucleotide strands are:

G1: 5'-TGG GGA CGG CGA CGG A GGG TTA GGG TTA GGG TTA GGG X-3', G2: 5'-CCG YCG CCG TCC CCA-3',

G3: 5'-TGG GGA CGG CGA CGG A GGG TTA GGG TTA GGG TTA GGG-T-3', G4: 5'-GGG TTA GGG TTA GGG TTA GGG-T-3'

G5: 5’-TGG GGA CGG CGA CGG TGT GGG TGG GTA GGG TGG GTA AX-3',

C1: 5'-TGG GGA CGG CGG A CCC TAA CCC TAA CCC TAA CCC Y-3',

C2: 5'-CCG XCG CCG TCC CCA-3'

C3: 5'-TGG GGA CGG CGG A CCC TAA CCC TAA CCC TAA CCC T-3'

C4: 5'-CCC TAA CCC TAA CCC TAA CCC T-3'

DS1: 5'-CCG TCG CCG TCC CCA-3', DS2: 5'-TGG GGA CGG CGA CGG-3',

( $\mathrm{X}$ is T-Atto 550 and $\mathrm{Y}$ is T-Atto $647 \mathrm{~N}$ ).

Annealing of the two oligonucleotide strand pairs (G1 or G5 \& G2; C1 \& C2) were carried out in a buffer containing $20 \mathrm{mM}$ Tris- $\mathrm{HCl}, 50 \mathrm{mM} \mathrm{NaCl}$ at $\mathrm{pH}$ 7.5. For annealing, the elongated strand (G1, G5 or C1) was mixed with the complementary strand (G2 or C2) in a molar ratio of 1:1 (both at $1 \mu \mathrm{M}$ after mixing) and annealed by first heating at $95^{\circ} \mathrm{C}$ for $5 \mathrm{~min}$, followed by gradually cooling down to room temperature at a rate of $-0.5^{\circ} \mathrm{C} / \mathrm{min}$ using a thermocycler. The buffer solution used in the measurements contains 20 $\mathrm{mM}$ Tris- $\mathrm{HCl}(\mathrm{pH} 7.4)$ and was filtered by a $0.45 \mu \mathrm{m}$ sterile Whatman Puradisc 30 syringe filter. After dilution, smFRET measurement were carried out at a DNA concentration of $\sim 50 \mathrm{pM}$, and the $\mathrm{NaCl}$ 
concentration was $\sim 2.5 \mu \mathrm{M}$ for the measurements in buffer and with $\alpha$-Syn monomers. For the measurements with $\alpha$-Syn aggregates, the $\mathrm{NaCl}$ concentration was up to $20 \mathrm{mM}$.

\section{Expression and purification of $\alpha$-synuclein}

The expression and purification of $\alpha$-synuclein was carried out as described before. ${ }^{2,3}$ The plasmid was purchased from GenScript. The plasmid pT7-7 expressing human $\alpha$-synuclein was transformed into Escherichia coli strain BL21 (DE3). A single colony was picked and inoculated into $100 \mathrm{~mL}$ LB medium containing $150 \mu \mathrm{g} / \mathrm{mL}$ ampicillin and grown at $37{ }^{\circ} \mathrm{C}$ with shaking at $250 \mathrm{rpm}$ until the absorbance at 600 $\mathrm{nm}$ reached 0.8 . Induction was then carried out by adding $1 \mathrm{mM}$ IPTG (final concentration) and the culture was further grown under similar conditions for $3 \mathrm{~h}$. The cells were harvested, resuspended in $0.75 \mathrm{~mL}$ of buffer $(50 \mathrm{mM}$ Tris- $\mathrm{HCl}, \mathrm{pH} 7.5,10 \mathrm{mM}$ EDTA and $150 \mathrm{mM} \mathrm{NaCl})$ and frozen at $-80{ }^{\circ} \mathrm{C}$. Tubes containing frozen cells were placed in a boiling water bath for $7 \mathrm{~min}$ and the supernatant collected after centrifugation at maximum speed for min. Streptomycin sulfate $(136 \mu \mathrm{L} / \mathrm{mL}$ of supernatant) and glacial acetic acid $(228 \mu \mathrm{L} / \mathrm{mL}$ of supernatant) were added and centrifuged for $2 \mathrm{~min}$. Again, the supernatant was recovered and precipitated with ammonium sulfate (saturated ammonium sulfate at $4^{\circ} \mathrm{C}$ was used $1: 1, \mathrm{v} / \mathrm{v}$, with supernatant). The protein was collected as a precipitate by centrifugation and washed once with 1 $\mathrm{mL}$ of ammonium sulfate solution $\left(4^{\circ} \mathrm{C}, 1: 1\right.$, v/v, saturated ammonium sulfate and water). The washed pellet was resuspended in $900 \mu \mathrm{L}$ of $100 \mathrm{mM}$ ammonium acetate (to form a cloudy solution) and precipitated by adding an equal volume of ethanol at room temperature. Precipitation with ethanol was repeated once more. The pellet was resuspended in $100 \mathrm{mM}$ ammonium acetate and extensively dialyzed against $10 \mathrm{mM}$ Tris- $\mathrm{HCl}$ buffer, $\mathrm{pH}$ 7.4.

\section{Preparation of $\alpha$-Synuclein aggregates}

Lyophilized $\alpha$-Synuclein was dissolved in $20 \mathrm{mM}$ Tris- $\mathrm{HCl}$ buffer ( $\mathrm{pH} 7.4)$ in the presence of $10 \%$ ethanol and $100 \mathrm{mM} \mathrm{NaCl}$ at $37{ }^{\circ} \mathrm{C}$ without stirring for $6 \mathrm{~h}$, followed by incubation at room temperature to perform all the measurements.

\section{Single-molecule FRET (smFRET) measurements}

SmFRET measurements were carried using a confocal fluorescence microscope (MicroTime 200, PicoQuant) under freely diffusing conditions. The pulsed interleaved excitation (PIE) FRET technique was used to separate dually labeled from singly labeled species. ${ }^{4}$ Briefly, in the PIE FRET technique, both the donor and acceptor are alternatively excited by the laser pulse. First, a laser pulse of suitable wavelength excites the donor and then another laser pulse excites the acceptor independently from FRET after a certain time delay ( $50 \mathrm{~ns}, 20 \mathrm{MHz}$ repetition rate), allowing us to calculate the photon stoichiometry, $S$, which is the ratio of photons emitted after donor excitation and the sum of total photons emitted after donor and direct acceptor excitation. For a donor-only species, $S=1$, and for an acceptor-species only, $S$ $=0$. A green laser pulse at $560 \mathrm{~nm}(\mathrm{LDH}$ series, PicoQuant) and a red laser pulse at $635 \mathrm{~nm}(\mathrm{LDH}$ series, PicoQuant) are used to excite the donor Atto 550 and acceptor Atto $647 \mathrm{~N}$, respectively. A quad band dichroic mirror (ZT 405/488/561/640, Chroma) was used to reflect both the green and red laser light to the entrance port of the fluorescence microscope. Donor and acceptor fluorescence signals were separated to two different detection channels, first by using a dichroic mirror (FF 650 Di01, Semrock), followed by band pass filters FF 01-593/40 (Semrock) and FF 01-676/29 (Semrock). Two SPCM-AQR series single photon avalanche diodes (SPAD) were used as detection channels for the donor and acceptor fluorescence.

The peaks in the FRET efficiency histograms are related to conformations with different spatial separations, $R$, of the two attached dyes and thus different FRET efficiencies, $E$, as $E=R_{0}^{6} \cdot\left(R_{0}^{6}+R^{6}\right)^{-1}$. Of note, a broadening in the mid FRET ( 0.4-0.6) region is most likely due to the non-linearity of the correlation between $E$ and $R$ and not necessarily related to a broadening of the conformational distribution. The Förster radius, $R_{0}$, i.e. the distance at which $50 \%$ of the excited donor molecules will be deactivated; here, $R_{0}=6.5 \mathrm{~nm}$ for the fluorophores used, Atto 550 and Atto $647 \mathrm{~N} .{ }^{5,6}$ Peak assignment is based on 
literature reports and verified through circular dichroism (CD) spectroscopy (Figures S4, S5). ${ }^{7,8} \mathrm{We}$ assigned the peaks at $E \approx 0.9$ in the $E$-histograms to an antiparallel conformation, and at $E \approx 0.6$ to a parallel or hybrid conformational state. In the presence of $\alpha$-Syn aggregates, a low FRET efficiency peak emerges at $E \approx 0.3$, which corresponds to the unfolded conformation of the G4Q. ${ }^{7,8}$ The ratio of DNA and protein where effects are starting to be seen is about $1: 10^{6}$. Whereas the protein needs to fill all the space evenly of the sample cell to have an effect on the DNA, the DNA itself is at low concentration to ensure single-molecule conditions and has only contact to the protein in a very small volume element, hence lowering the effective concentration ratio.

\section{CD measurements}

The topology of G-quadruplexes and i-motifs can be determined by monitoring the positive or negative circular dichroism (CD) signals at specific wavelengths. The CD measurements were performed using a JASCO J-715 spectropolarimeter in $20 \mathrm{mM} \mathrm{Na} \mathrm{H}_{\mathrm{x}} \mathrm{PO}_{4}$-buffer at $\mathrm{pH} 7.5$ or in $20 \mathrm{mM}$ sodium acetate buffer at $\mathrm{pH} 5$, respectively. The concentration of the DNA construct was $20 \mu \mathrm{M}$. The annealing was carried out as described above. The molar ellipticity was calculated per nucleobase. The spectra were corrected by subtraction of the buffer spectrum and smoothed using a FFT-filter if necessary. For the Gquadruplex construct (G3 annealed with DS1) and the i-motif construct (C3 annealed with DS1), the spectrum of the double strand (DS2 annealed with DS1) was subtracted to better emphasize the CD spectrum of the neat G-rich and the corresponding C-rich sequence, assuming additivity of the CD spectra. This is shown by the comparison of G4 and C4 with the corresponding constructs (G3+DS1 \& C3+DS1) in Figures S4A and B. The peak positions of the G-quadruplex construct are consistent with the antiparallel basket conformation described in the literature. ${ }^{9,10}$ The structure of the partially folded telomeric $\mathrm{i}$-motif is less explored. Hence, temperature dependent $\mathrm{CD}$ data were recorded at $\mathrm{pH} 7.5$ (Figure S5A) and compared to the data at $\mathrm{pH} 5$ (Figure S5B). The comparison reveals a shift of the peak at 285 $\mathrm{nm}$ by about $10 \mathrm{~nm}$ at $25^{\circ} \mathrm{C}$, which is reduced to $5 \mathrm{~nm}$ at $80{ }^{\circ} \mathrm{C}$, indicating that both sequences unfold from different starting conformations to a similar end point structure, the fully unfolded state, at high temperature. Hence, we can safely conclude that the structure at $\mathrm{pH} 7.5$ recorded by smFRET with an energy transfer efficiency of $E \approx 0.6$ corresponds to the partially folded i-motif. Figure S6 shows smFRET measurements of the i-motif construct at $\mathrm{pH} 5$ to proof that the high-FRET efficiency of $\sim 0.9$ corresponds in fact to the fully folded i-motif as described by Jungkweon Choi et al. ${ }^{11}$ for the acidic $\mathrm{pH}$.

\section{Dynamic Light Scattering (DLS)}

DLS experiments were carried out by means of a Zetasizer Nano $\mathrm{S}$ from Malvern Instruments Limited (Malvern Worcs, U.K.). The instrument is equipped with a He-Ne laser operating at 633nm. The temperature was set at $25^{\circ} \mathrm{C}$. Each distribution function is the results of 15 accumulations. 


\section{Supporting Figures}
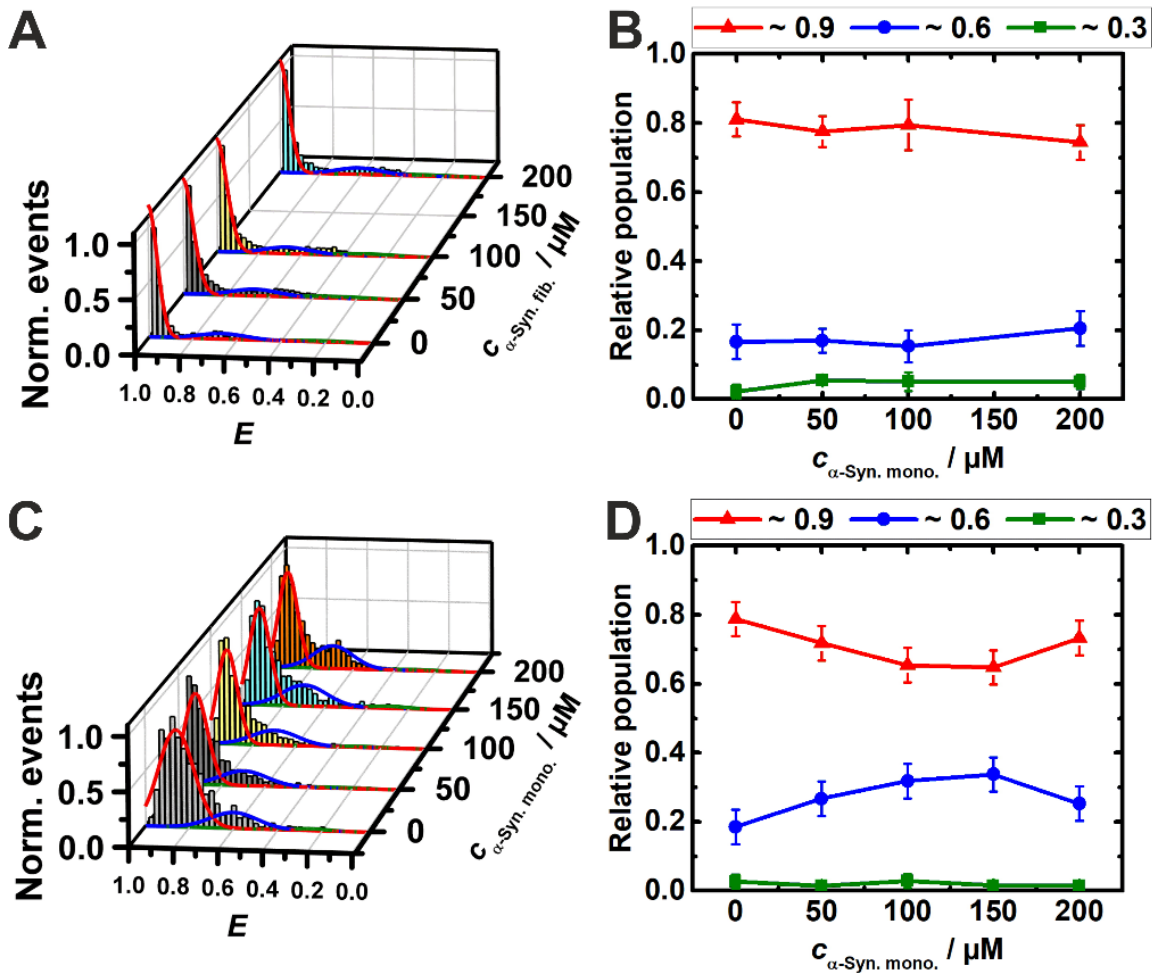

Figure S1: FRET efficiency histogram (A) and relative population of conformers (B) of the G4Q in buffer with increasing concentration of $\alpha$-Syn monomers. The solution in (C) and (D) contained additionally 30 wt\% Ficoll PM 70.
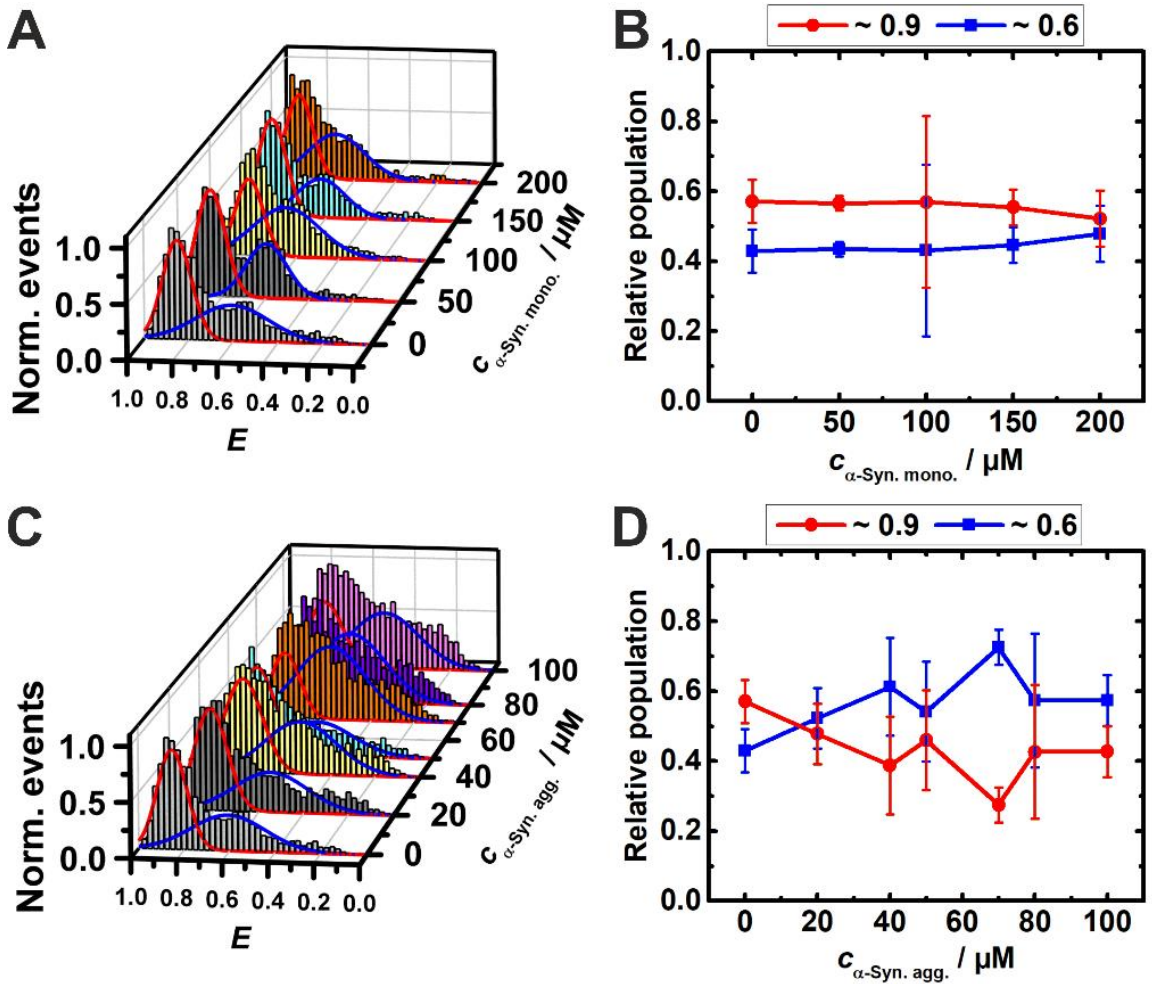

Figure S2: FRET efficiency histograms and relative population of conformers of the i-motif in buffer containing $30 \mathrm{wt} \%$ Ficoll PM 70 with increasing concentration of $\alpha$-Syn monomers (A \& B) or $\alpha$-Syn aggregates (C \& D). 


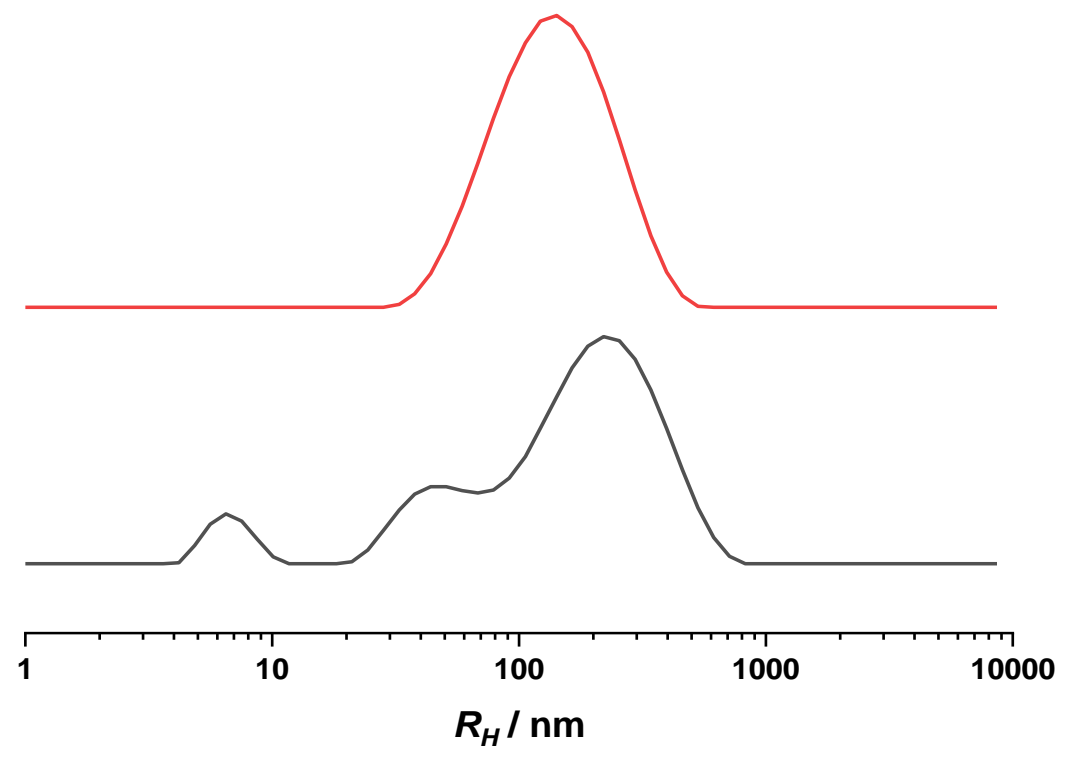

Figure S3: Hydrodynamic radius distribution functions for $50 \mu \mathrm{M}$ (black line) and $100 \mu \mathrm{M}$ (red line) $\alpha$ Synuclein solutions. The experiments were carried out at $25{ }^{\circ} \mathrm{C}$ after treatment of the solution at $40{ }^{\circ} \mathrm{C}$ for $6 \mathrm{~h}$.
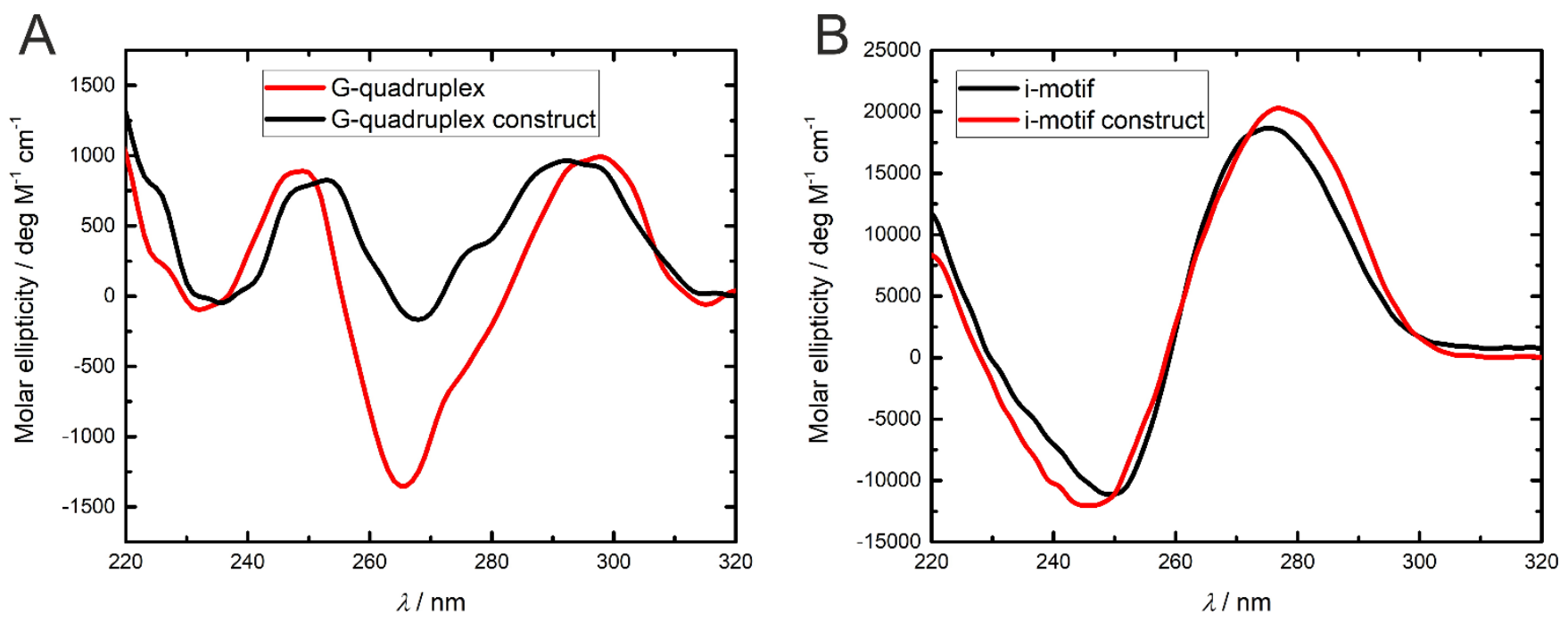

Figure S4: $\mathrm{CD}$ measurements of (A) the G-quadruplex and the corresponding construct in buffer containing $20 \mathrm{mM}$ Tris/ $\mathrm{HCl} \mathrm{pH} 7.5$ and $15 \mathrm{mM} \mathrm{NaCl}$ as well as (B) the i-motif and the corresponding construct in buffer containing $20 \mathrm{mM} \mathrm{Na}_{x} \mathrm{H}_{\mathrm{x}} \mathrm{PO}_{4}$-buffer $\mathrm{pH}$ 7.5. The spectrum of the double strand part was subtracted from the spectra of the G-quadruplex construct as well as the i-motif construct. 

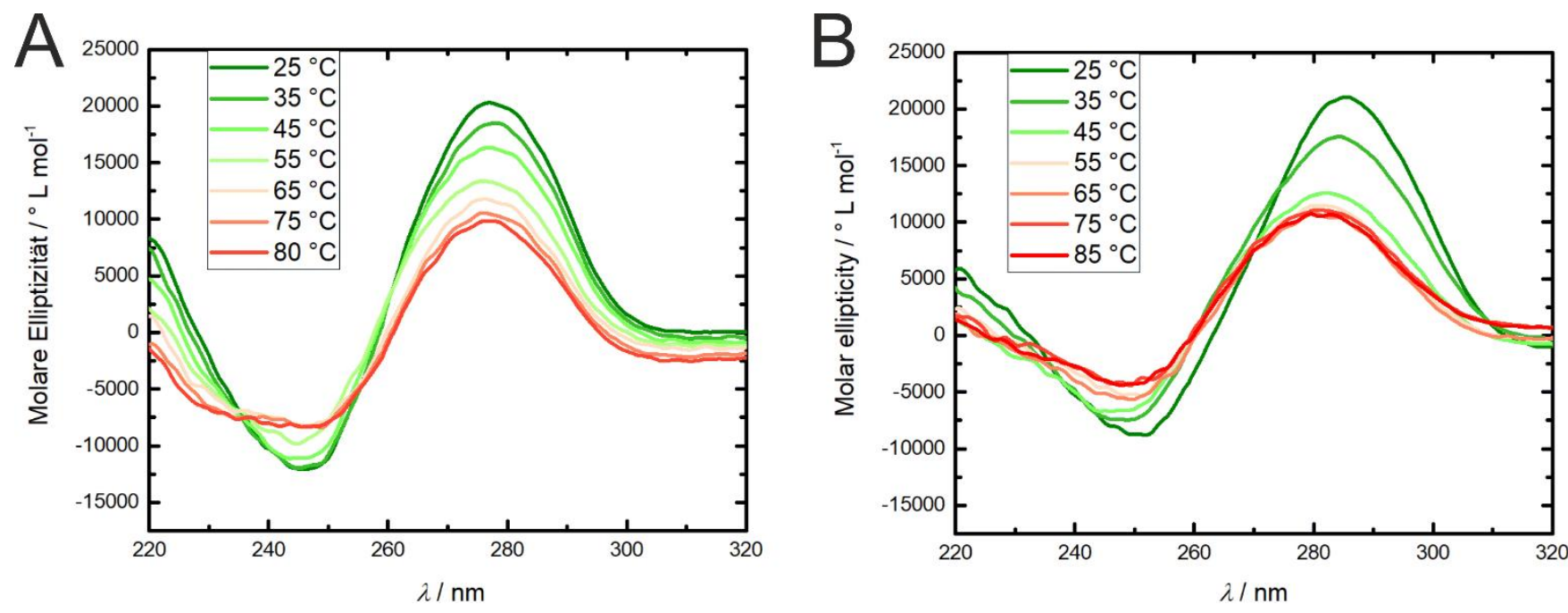

Figure S5: Temperature dependent $\mathrm{CD}$ measurements of the i-motif construct in buffer containing 20 $\mathrm{mM} \mathrm{Na}{ }_{x} \mathrm{H}_{\mathrm{x}} \mathrm{PO}_{4}$ at $\mathrm{pH} 7.5$ (A) and $20 \mathrm{mM}$ sodium acetate buffer at $\mathrm{pH} 5$ (B).

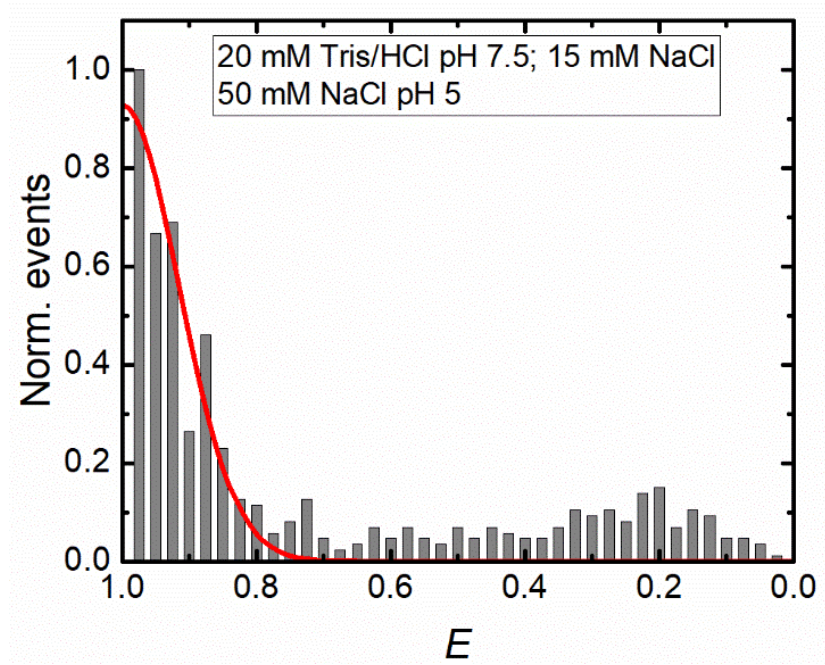

Figure S6: SmFRET measurement of the i-motif construct in $20 \mathrm{mM}$ sodium acetate buffer at $\mathrm{pH} 5$ and at $T=25^{\circ} \mathrm{C}$. 
A

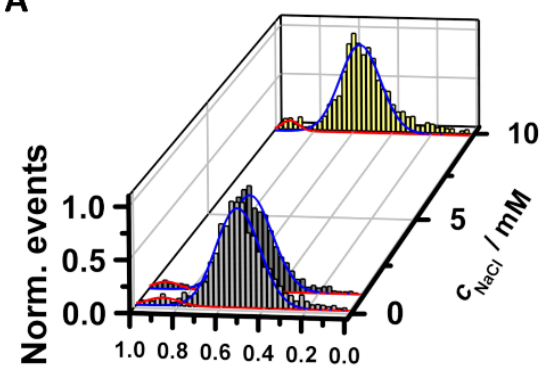

$E$

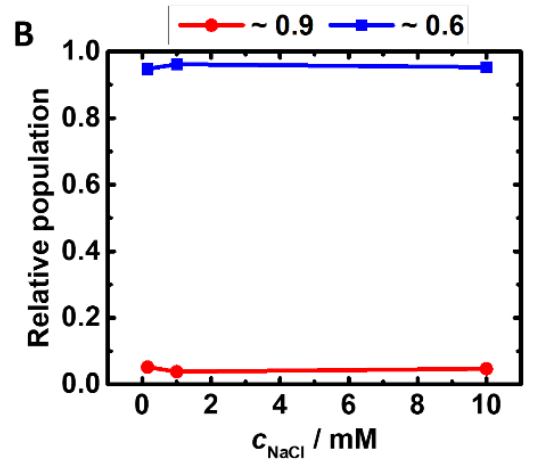

C

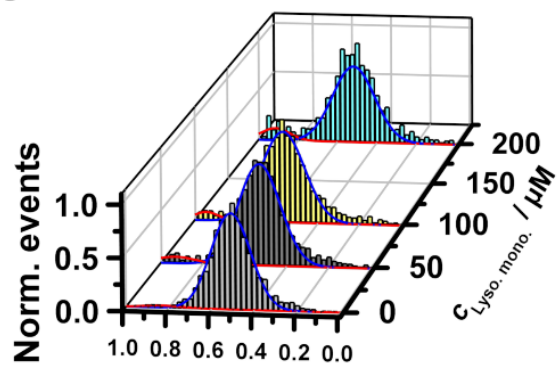

E

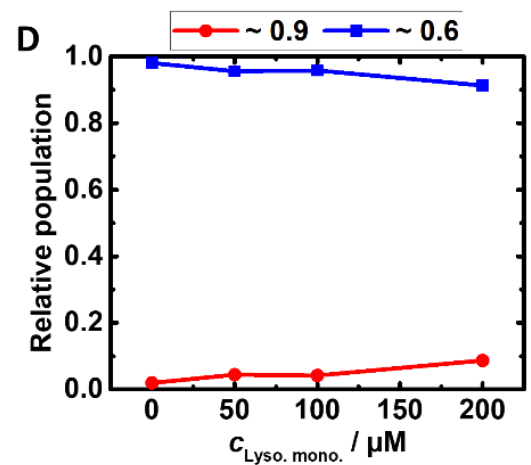

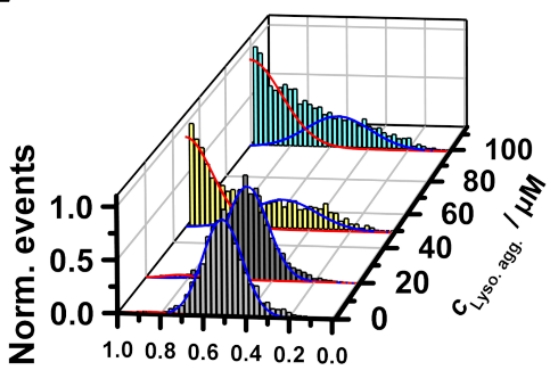

$E$

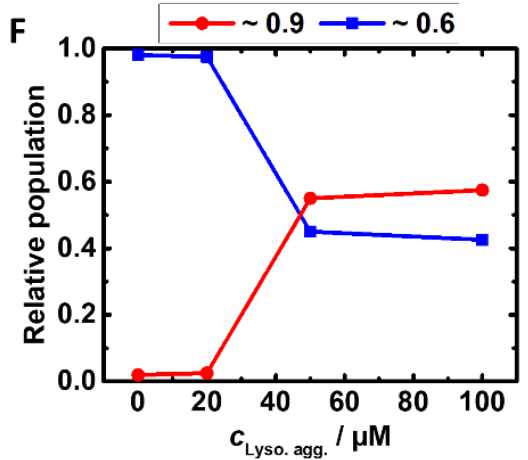

Figure S7: SmFRET histograms and relative population of conformers of the i-motif construct in $20 \mathrm{mM}$ Tris/ $\mathrm{HCl}, \mathrm{pH} 7.5, T=25{ }^{\circ} \mathrm{C}$, further containing (A, B) NaCl, $(\mathrm{C}, \mathrm{D})$ lysozyme monomers or $(\mathrm{E}, \mathrm{F})$ lysozyme aggregates.

\section{telomeric G-quadruplex}

A

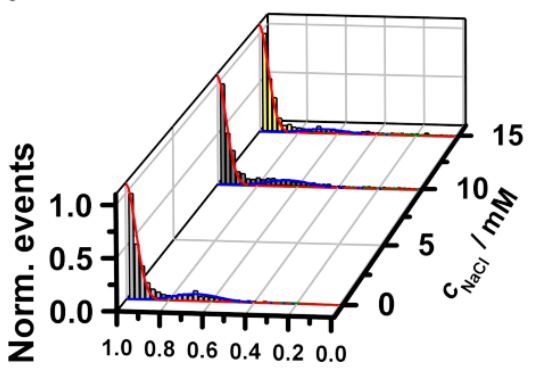

$E$

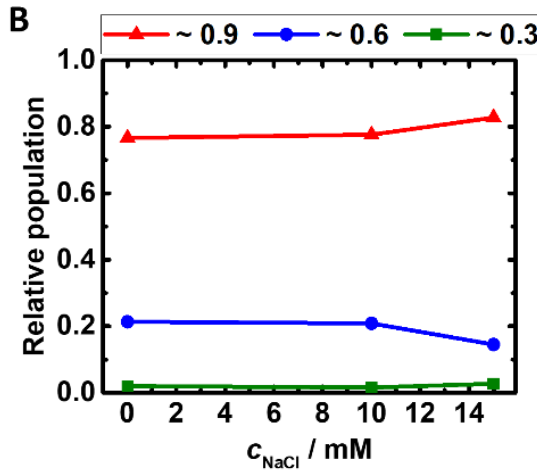

C

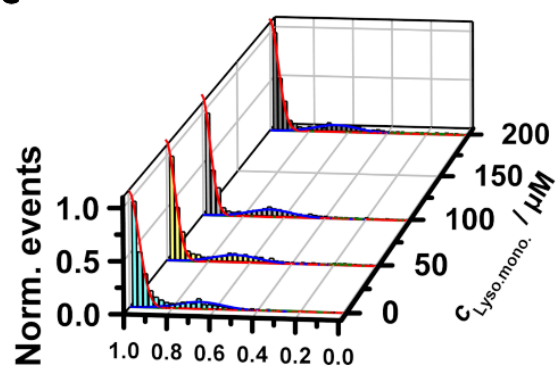

E

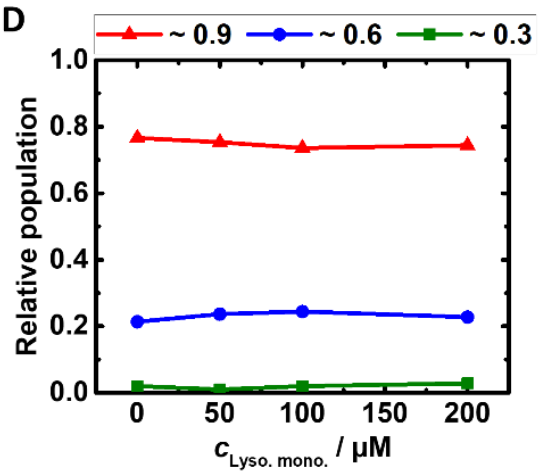

E

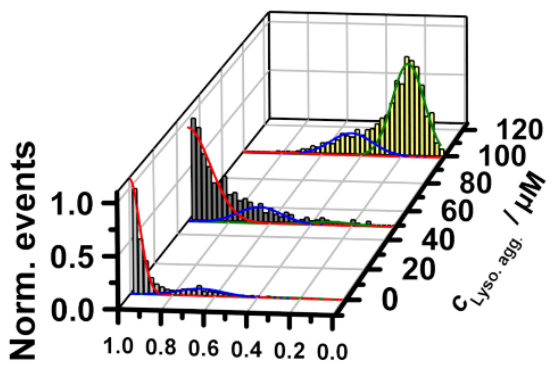

E

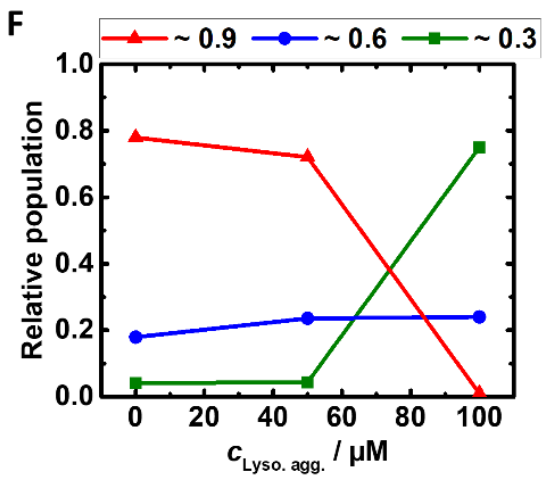

Figure S8: SmFRET histograms and relative population of conformers of the telomeric G-quadruplex construct in $20 \mathrm{mM}$ Tris $/ \mathrm{HCl}, \mathrm{pH} 7.5$, at $T=25{ }^{\circ} \mathrm{C}$, further containing (A, B) $\mathrm{NaCl},(\mathrm{C}, \mathrm{D})$ lysozyme monomers or $(\mathrm{E}, \mathrm{F})$ lysozyme aggregates. 
Figures S7 and S8 show additional smFRET measurements at different concentrations of $\mathrm{NaCl}$ and of monomeric and aggregated lysozyme. The higher $\mathrm{NaCl}$ concentrations have no effect on the conformation of the i-motif and only a slight stabilization effect on the G4Q in the physiological range of 10 to $15 \mathrm{mM}$ compared to the neat buffer conditions $(\sim 2.5 \mu \mathrm{M})$. Lysozyme monomers impose no effect both on the conformation of the i-motif and the G4Q, similar to the results for monomeric $\alpha$-Syn (Figures S1 A\&B). The effect of lysozyme monomers on the i-motif differs from that of the $\alpha$-Syn monomers (Figures 3 A\&B), which is most likely due the missing flexibility of the globular lysozyme compared to the IDP $\alpha$ Syn, which, owing to its intrinsically high conformational dynamics, allows a better matching of opposite charged patches for the IDP and the i-motif. In turn, lysozyme aggregates impose a similar effect as the $\alpha$-Syn aggregates on both, the $\mathrm{i}$-motif and $\mathrm{G} 4 \mathrm{Q}$, supporting the notion that secondary and tertiary structure formation is responsible for the different effects of similarly charged proteins.

\section{c-myc promoter G-quadruplex}
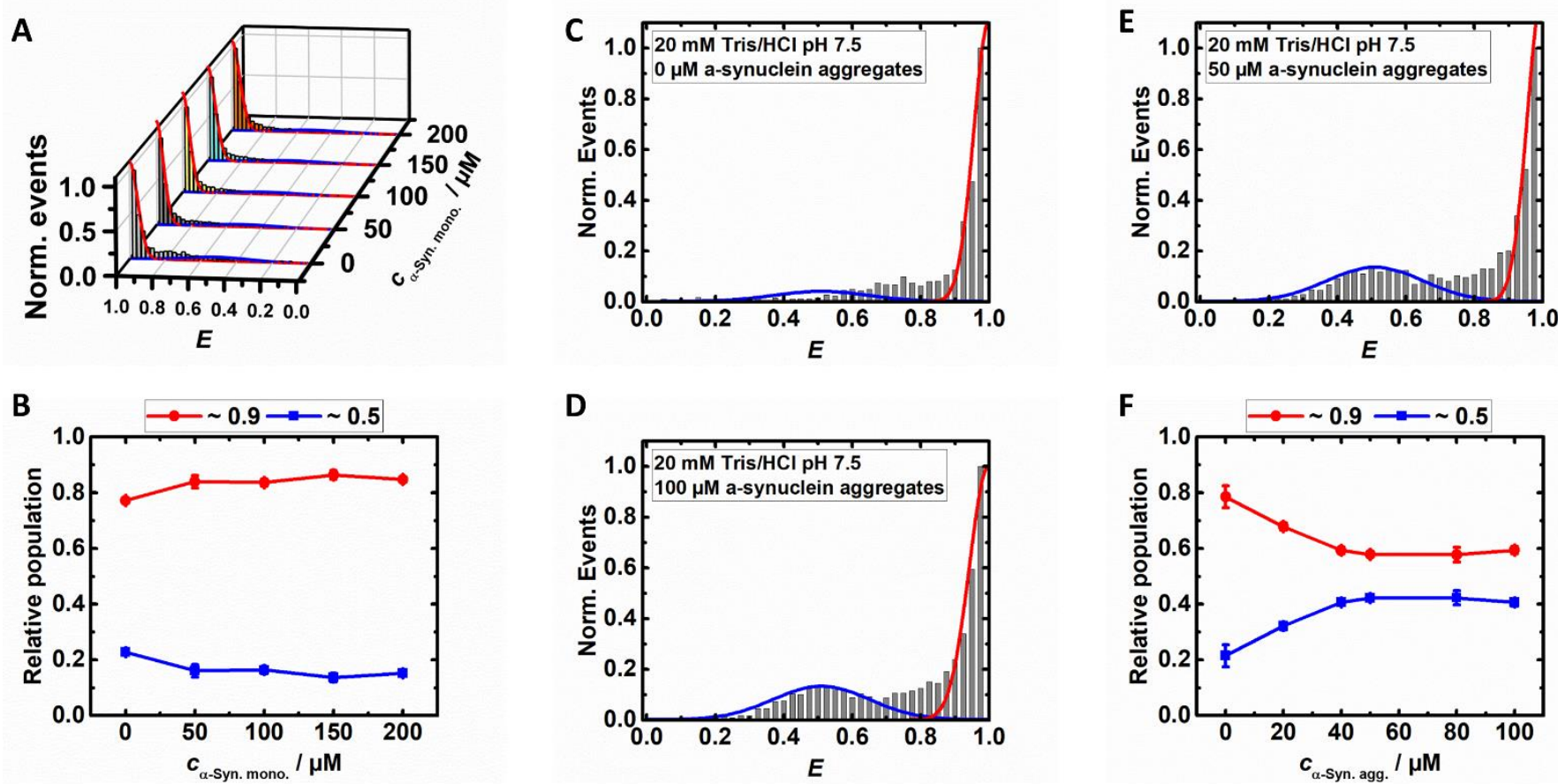

Figure S9: SmFRET histograms and relative population of conformers of the c-Myc G-quadruplex construct (G5 + G2) in $20 \mathrm{mM}$ Tris/ $\mathrm{HCl}, \mathrm{pH} 7.5$, at $T=25^{\circ} \mathrm{C}$, further containing (A, B) increasing concentrations of $\alpha$-synuclein monomers or $(\mathrm{C}, \mathrm{D}, \mathrm{E}, \mathrm{F})$ increasing concentrations of $\alpha$-synuclein aggregates.

Myc is a family of regulator genes and proto-oncogenes that code for transcription factors. Figure S9 shows the effect of monomeric and aggregated $\alpha$-synuclein on a G-quadruplex construct of the c-Myc promoter region (c-MycG4), which was characterized by Liu et al. ${ }^{12}$ This sequence is known to suppress c-Myc expression by forming a G-quadruplex. ${ }^{13}$ Formation of the G4 can be modulated by drug binding, hence establishing these DNA structures as promising targets in cancer therapy. We included the double strand part in the c-MycG4 sequence for the second dye of the FRET-pair to be able to directly comparable these data with the G4Q construct. Depending on the conditions, two conformations could be distinguished in smFRET experiments, one with high FRET efficiency, $E$, and a second one with a center at $E \approx 0.5$. Hence, a two Gaussian fit model was used to determine the ratio of the two conformers. Addition of monomeric $\alpha$-synuclein had no effect on the conformational distribution of the c-mycG4 (Fig. S9B), which is a similar result compared to the G4Q data (Fig. S1B). In contrast, aggregates of $\alpha$ synuclein had an impact on the distribution of conformers, leading to a marked increase of the population with $E \approx 0.5$ (Fig. S9F). Literature reports a parallel conformation for c-Myc G-quadruplexes in $\mathrm{KCl}$ solution, ${ }^{14}$ so that the high-FRET peak can be attributed to a parallel conformation, while $E \approx 0.5$ 
represents most probably an essentially unfolded conformation. This would also be in accordance with the unfolding effect imposed by $\alpha$-synuclein aggregates on the G4Q structure. In conclusion, aggregated $\alpha$-synuclein impacts also the structure of c-MycG4, the effect is less pronounced compared to that on the G4Q structure, however, which might be due to a more stable G4 structure.

\section{References}

1. Baltierra-Jasso, L. E.; Morten, M. J.; Laflör, L.; Quinn, S. D.; Magennis, S. W. Crowding-Induced Hybridization of Single DNA Hairpins. J. Am. Chem. Soc. 2015, 137, 16020-16023.

2. Volles, M. J.; Lansbury, P. T. Relationships between the sequence of $\alpha$-synuclein and its membrane affinity, fibrillization propensity, and yeast toxicity. J. Mol. Biol. 2007, 366, 1510-1522.

3. Shaltiel-Karyo, R.; Frenkel-Pinter, M.; Egoz-Matia, N.; Frydman-Marom, A.; Shalev, D. E.; Segal, D.; Gazit, E. Inhibiting a-Synuclein Oligomerization by Stable Cell Penetrating b-Synuclein Fragments Recovers Phenotype of Parkinson's Disease Model Flies. PLoS One, 2010, 5, e13863.

4. Ruettinger, S.; Buschmann, V.; Kraemer, B.; Orthaus, S.; Koberling, F. FRET analysis with Pulsed Interleaved Excitation (PIE) using the MicroTime 200. Appl. note, PicoQuant GmbH, 2013.

5. Arns, L.; Winter, R. Liquid-liquid phase separation rescues the conformational stability of a DNA hairpin from pressure-stress. Chem. Comm. 2019, 55, 10673-10676.

6. Patra, S.; Anders, C.; Erwin, N.; Winter, R. Osmolyte Effects on the Conformational Dynamics of a DNA Hairpin at Ambient and Extreme Environmental Conditions. Angew. Chemie. Int. Ed. 2017, 56, 5045-5049.

7. Ying, L.; Green, J. J.; Li, H.; Klenerman, D.; Balasubramanian, S. Studies on the structure and dynamics of the human telomeric $\mathrm{G}$ quadruplex by single-molecule fluorescence resonance energy transfer. Proc. Natl.Acad. Sci. USA 2003, 100, 14629-14634.

8. Tippana, R.; Hwang, H.; Opresko, P. L.; Bohr, V. A.; Myong, S. Single-molecule imaging reveals a common mechanism shared by G-quadruplex-resolving helicases. Proc. Natl.Acad. Sci. USA 2016, 113, 8448-8453.

9. del Villar-Guerra, R.; Trent, J. O.; Chaires, J. B. G-quadruplex secondary structure from circular dichroism spectroscopy. Angew. Chemie - Int. Ed. 2018, 57, 7171-7175.

10. Miller, M. C.; Buscaglia, R.; Chaires, J. B.; Lane, A. N.; Trent, J. O. Hydration Is a Major Determinant of the G-Quadruplex Stability and Conformation of the Human Telomere 3' Sequence of $\mathrm{d}\left(\mathrm{AG}_{3}\left(\mathrm{TTAG}_{3}\right)_{3}\right)$. J. Am. Chem. Soc. 2010, 132, 17105-17107.

11. Choi, J.; Kim, S.; Tachikawa, T.; Fujitsuka, M.; Majima, T. pH-Induced Intramolecular Folding Dynamics of i-Motif DNA. J. Am. Chem. Soc. 2011, 133, 16146-16153.

12. Liu, L.; Ma, C.; Wells, J. W.; Chalikian, T. V. Conformational Preferences of DNA Strands from the Promoter Region of the c-Myc Oncogene. J. Phys. Chem. B 2020, 124, 751-762.

13. Siddiqui-Jain, A.; Grand, C. L.; Bearss, D. J.; Hurley, L. H. Direct evidence for a G-quadruplex in a promoter region and its targeting with small molecules to repress c-MYC transcription. Proc. Natl. Acad. Sci. U.S.A. 2002, 99, 11593-11598.

14. Ambrus, A.; Chen, D.; Dai, J.; Jones, R. A.; Yang, D. Solution Structure of the Biologically Relevant G-Quadruplex Element in the Human c-Myc Promoter. Implications for G-Quadruplex Stabilization. Biochemistry 2005, 44, 2048-2058. 